\title{
Cystic lymphangioma of the jejunal mesentery in a young adult: A case report with review of literature
}

\author{
Dr Aarti B Bhattacharya ${ }^{1}$, Dr Shamima ${ }^{2}$, Dr Rajeev K Agarwal $^{3}$ \\ ${ }^{l}$ Professor, Department of Pathology, Hind Institute of Medical Sciences, Safedabad, Barabanki, U.P., India \\ ${ }^{2}$ Assistant Professor, Department of Pathology, Hind Institute of Medical Sciences, Safedabad, Barabanki, U.P., \\ India \\ ${ }^{3}$ Associate Professor, Department of Surgery, Hind Institute of Medical Sciences, Safedabad, Barabanki, U.P., \\ India
}

\begin{abstract}
Cystic lymphangioma of the small bowel mesentery is a rare manifestation of an intraabdominal tumor particularly in adults. ${ }^{1,2,3}$ The clinical features of intra abdominal lymphangioma are diverse ranging from an asymptomatic abdominal tumor to symptoms of acute abdomen, thus a mass may be discovered incidentally during abdominal examination for an unrelated illness. We hereby present the case of a young adult male with cystic lymphangioma of the jejunal mesentery who presented with an abdominal mass and history of sitophobia.
\end{abstract}

\section{Case report:}

A 20 years old male presented with the complaint of postprandial severe abdominal pain since ten days which was not responding to analgesics. He had sitophobia and significant weight loss. On clinical examination there was disproportionate abdominal sign. $\mathrm{X}$ ray abdomen revealed multiple fluid and gas shadows. No abnormal free gas was seen under both domes of diaphragm. Contrast CT Scan abdomen revealed a large hyperintense mesenteric mass at the junction of jejunum and ileum. Rest other routine investigations including hematological and biochemical tests were within normal limits. He had past history of surgery for appendicitis and Meckel's diverticulitis. He underwent elective laparotomy and a large yellowish pink cystic lesion was found in the mesentery at the junction of jejunum and ileum. It was carefully resected along with the segment of jejunum and sent for histopathology. Postoperatively there was no evidence of infection in the drain, which was removed on $8^{\text {th }}$ day. He was allowed to have food orally on $9^{\text {th }}$ day, but after eating he developed severe abdominal pain. On examination there was mild tenderness in the left hypochondrium. At emergency laparotomy on the same day, the whole small bowel from $10 \mathrm{~cm}$ distal to ileocaecal junction up to the jejunum was found to be gangrenous. Resection and anastomosis was performed. Post operative stay in the hospital was uneventful. The patient was advised regarding long term follow up that is required to detect recurrence.

The surgical specimen obtained from the first surgery consisted of a large yellowish pink cystic mass measuring $19 \times 11 \times 10 \mathrm{~cm}$ attached to the mesentery and to the wall of jejunal segment measuring $18 \mathrm{~cm}$ in length. The mass showed multiple cystic spaces of varying sizes, cut surface was partly cystic and partly solid. Cystic spaces were filled with milky fluid. Biochemical studies of the fluid revealed high levels of cholesterol and triglycerides. Histological examination revealed multiple dilated channels lined by flattened endothelial cells and separated by collagenous tissue containing small collections of lymphocytes. The dilated lymphatic channels had no connection to normal adjacent lymphatics. The final diagnosis of mesenteric cystic lymphangioma was given. The second operation surgical specimen showed gangrenous changes due to lymphatic obstruction.
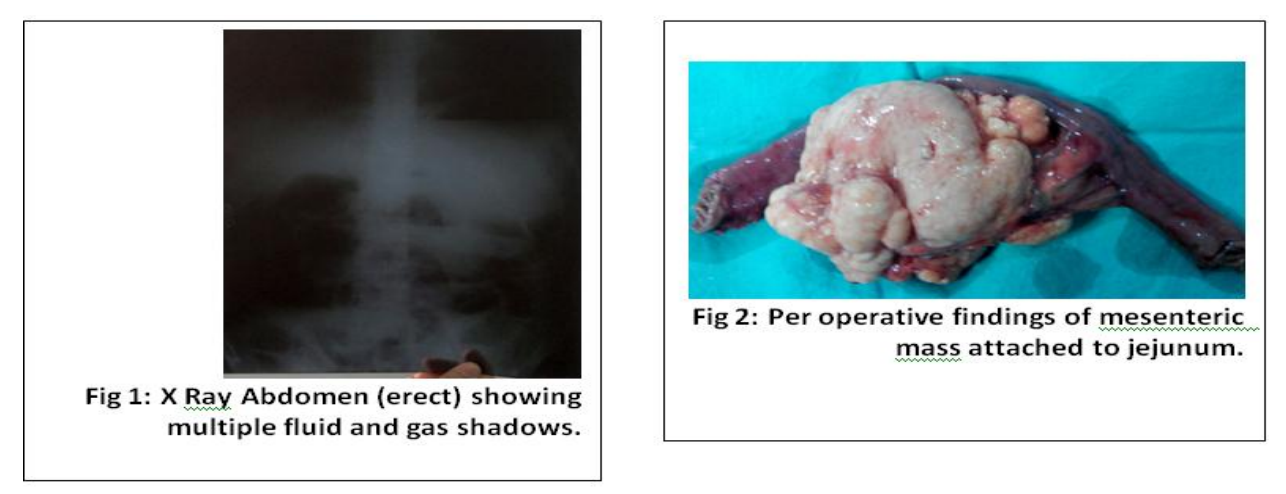


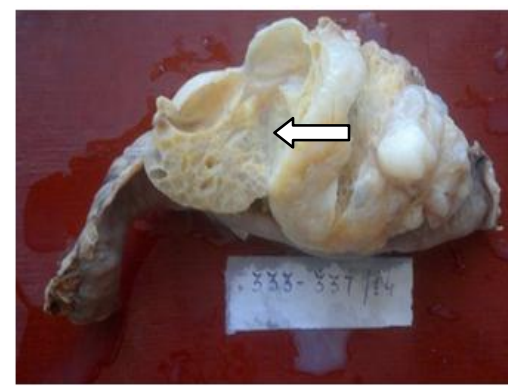

Fig 3: Specimen of mesenteric cystic lymphangioma filled with chylous fluid

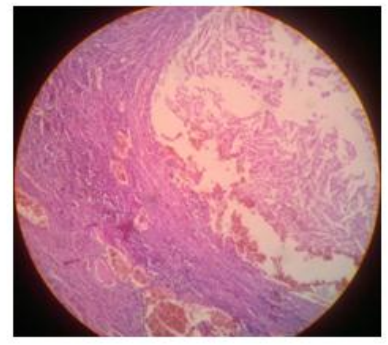

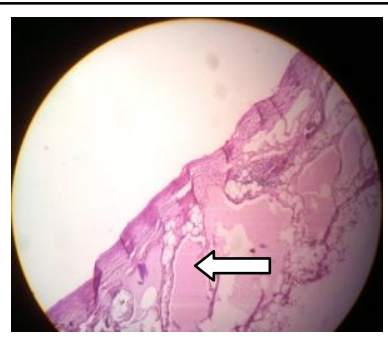

Fig 4: Mic. H\&E (4X) mesenteric Lymphangioma showing multiple dilated lymphatic spaces filled with chylous fluid

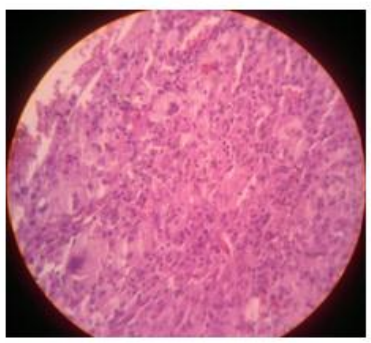

Fig 5, 6: Mic. H\&E (10X, 40X) showing dilated lymphatic spaces, cholestrol clefts, foam cells, foreign body type giant cells and vascular congestion.

\section{Discussion:}

Lymphangiomas are uncommon benign tumors occurring mainly in children. Almost $90 \%$ of these occur in children younger than 3 years of age. ${ }^{1,2}$ In the children the most common sites are head and neck and axillary regions. Intra -abdominal lymphangiomas are very rare, accounting for $<1 \%$ of all the lymphangiomas. ${ }^{4,5}$ Thus benign mesenteric lymphangioma accounts for in between 1 in 1,00,000 to 1 in 2,50,000 hospital admissions. ${ }^{6,7}$

Lymphangioma can occur throughout the body but the commonest site is neck where it is called as cystic hygroma. Within the abdomen the commonest site is mesentery, other sites being omentum, retroperitoneum, mesocolon and rarely pancreas. ${ }^{5,8}$,

The etiology of lymphangioma is unclear. Congenital developmental defects of the lymphatics have been proposed. Proliferation and dilatation of blind lymphatic sacks lacking proper connection with the venous system results in cystic lymphangioma. ${ }^{4}$ The congenital etiological theory is supported by the fact that most of the cases manifest in childhood. Other potential causes are trauma to abdomen, lymphatic disease and obstruction, inflammatory processes, surgery and radiation therapy which may lead to secondary formation of such a tumor. ${ }^{1}$

Clinically intra-abdominal lymphangiomas may be asymptomatic usually when small, or may give rise to symptoms of acute abdomen as it enlarges. A gradual increase in abdominal girth, sensation of fullness and vague abdominal pain may point to this rare benign condition. Pain may be dull as in chronic abdominal pain or it may be more acute due to torsion of the cyst, hemorrhage in the cyst, infection and rupture of the cyst. ${ }^{10}$ An enlarged cyst may compress the surrounding structures or cause intestinal obstruction. They often behave in an aggressively invasive manner and can even grow to enormous size involving the surrounding structures therefore resection of the adjacent organs may be required to accomplish complete excision of the cyst.

Histopathology of the specimen is the gold standard for diagnosis. ${ }^{11}$ No blood test confirms the diagnosis. Radiological investigations like USG and CT are insufficient to provide a definite clue. MRI aids in the diagnosis as it defines the origin of the mass. Jain et al reported establishing a preoperative definite diagnosis of an intra-abdominal lymphangioma using multi slice spiral CT. ${ }^{12}$

Mesenteric Lymphangioma has to be differentiated from multi-cystic mesothelioma, lymphangiomyoma and cavitatory mesenteric tuberculous lymph node. ${ }^{13}$ Immunohistochemicaly Fc VIII related $\mathrm{Ag}$ and $\mathrm{D}_{2-40}$ in lymphangioma, Calretinin in multi-cystic mesothelioma and HMB-45 is positive in lymphangiomyoma. ${ }^{14}$

Treatment of intra abdominal lymphangioma is surgical resection and anastomosis. Till now open surgery was being performed, however laparoscopic resection has been reported in few instances. ${ }^{15}$ During surgery occasionally resection of adjacent structures like small bowel may be 
warranted when the lymphangioma involves the bowel or its important vessels. In some cases it precludes total cyst removal because of its proximity to vital structures and may then result in recurrence or post operative complications. In these cases alcohol, bleomycin and $\mathrm{O} \mathrm{K}-432$ have been used alternatively and proved to be successful. ${ }^{16}$

Histologically lymphangiomas are classified into three types : 1. simple-capillary, 2. cavernous and 3. cystic lymphangioma. Simple - capillary lymphangioma is usually located superficially in the skin comprising small thin walled lymphatics. The cavernous lymphangioma consists of larger lymphatics having connection with adjacent normal lymphatics. The cystic lymphangioma consists of lymphatic spaces of various sizes that may contain serous, chylous, bloody or purulent fluid but it has no connection with the normal adjacent lymphatics. ${ }^{16,17}$ In our case the lymphatic spaces contained chylous fluid.

\section{Conclusion:}

Mesenteric lymphangioma is very rare and may be an incidental finding. It may present as an abdominal mass or as an acute abdomen necessitating emergency surgery. Therefore it should be considered in the differential diagnosis of a mass in abdomen as well as acute abdomen.

\section{References:}

[1]. Chen CW, Hsu SD, Lin CH, Cheng MF, Yu JC. Cystic lymphangioma of the jejunal mesentery in an adult: a case report. World J Gastroenterol. 2005;11:5084-5086.

[2]. Rieker RJ, Quentmeier A, Weiss C, Kretzschmar U, Amann K, Mechtersheimer G, Bläker H, Herwart OF. Cystic lymphangioma of the small-bowel mesentery: case report and a review of the literature.Pathol Oncol Res. 2000;6:146-148

[3]. Hanagiri T, Baba M, Shimabukuro T, et al. Lymphangioma in the small intestine: report of a case and review of the Japanese literature. Surg Today. 1992;22:363-7.

[4]. Weeda VB, Booij KA, Aronson DC. Mesenteric cystic lymphangioma: a congenital and an acquired anomaly? Two cases and a review of the literature. J Pediatr Surg. 2008 Jun;43(6):1206-8. doi: 10.1016/j.jpedsurg.2008.01.075.

[5]. Stein M, Hsu RK, Schneider PD, Ruebner BH, Mina Y. Alcohol ablation of a mesenteric lymphangioma. J Vasc Interv Radiol. 2000 Feb;11(2 Pt 1):247-50.

[6]. H Chim, E Chuwa, Y-P Chau, PKH Chow. Gastrointestinal: Mesenteric cystic lymphangioma. Journal of Gastroenterology and Hepatology. Volume 21, Issue 5, page 916,May 2006

[7]. W J Campbell, S T Irwin, J D Biggart. Gut 1991 32: 1568. Benign lymphangioma of the jejunal mesentery: an unusual cause of small bowel obstruction.

[8]. Milliaras S., Trygonis S., Papandoniou A., Kalamaras S., Trygonis C., Kiskinis D. Mesenteric cyst of the discending colon: a case report. Acta Chir Belg. 2006;106:714-716.

[9]. Koenig TR, Loyer EM, Whitman GJ, Raymond AK, Charnsangavej C. Cystic lymphangioma of the pancreas. AJR Am J Roentgenol. 2001;177:1090.

[10]. Seki H, Ueda T, Kasuya T, et al. Lymphangioma of the jejunum and mesentery presenting with acute abdomen in an adult. $\mathrm{J}$ Gastroenterol. 1998;33:107-11.

[11]. Solomon Akwei, Neil Bhardwaj, and Paul D Murphy. Benign mesenteric lymphangioma presenting as acute pancreatitis: a case report. Cases J. 2009; 2: 9328. Published online Dec 16, 2009. doi: 10.1186/1757-1626-2-9328. PMCID: PMC2803988

[12]. Jain S, Upreti L, Bhargava S K, Gupta R, Gupta P K. Mesenteric lymphangioma: diagnosis by multislie spiral CT (Letter). Indian J Radiol Imaging (serial online) 2002 (cited 2014 april 17); 12:580-2

[13]. Rosai J, Ackerman LV. Rosai and Ackerman's surgical pathology. 10th ed. Vol 2, Edinburgh: Mosby; 2011. Chapter 26, Page 2248 .

[14]. Worapop Suthiwartnarueput, Siriphut Kiatipunsodsai, Amolchaya Kwankua, and Utairat Chaumrattanakul. Lymphangioma of the small bowel mesentery: A case report and review of the literature. World J Gastroenterol. Nov 21, 2012; 18(43): 6328-6332. Published online Nov 21, 2012. doi: 10.3748/wjg.v18.i43.6328. PMCID: PMC3501784

[15]. Losanoff $\mathrm{JE}^{1}$, Kjossev KT. Mesenteric cystic lymphangioma: unusual cause of intra-abdominal catastrophe in an adult. Int J Clin Pract. 2005 Aug;59(8):986-7.

[16]. Jun Chul Chung and Ok Pyung Song. Cystic lymphangioma of the jejunal mesentery presenting with acute abdomen in an adult. Can J Surg. Dec 2009; 52(6): E286-E288. PMCID: PMC2792400

[17]. A Nejmeddine, A Sonia, H Sondes, B Salah, B Issam. Mesenteric cystic lymphangioma. The Internet Journal of Surgery. 2008 Volume 19 Number 1 